\title{
Research on Safety Measures of Shallow-buried and Undercut Tunnel
}

\author{
LIU jian-jun ${ }^{1}$, LIU Xue-ying ${ }^{1 *}$, FAN Zhong-jing ${ }^{2}$, and Yang Bao-feng ${ }^{3}$ \\ ${ }^{1}$ Zhejiang University of Water Resources and Electric Power, 310018 Hangzhou, Zhejiang China \\ ${ }^{2}$ China Railway Tunnel Bureau Group Construction Co., Ltd, 530000 Quzhou, Zhejiang China \\ ${ }^{3}$ Ningbo Longyuan Shenghong Ecological Construction Engineering Co., Ltd, 315327 Ningbo, Zhejiang, China
}

\begin{abstract}
The application of mine method shallow burying and undercutting method in subway and tunnel engineering is becoming more and more significant, but shallow burying and undercutting method also has its own limitations: the shallow burying and undercutting method can easily cause the subsidence of nearby surface, there is a security threat to surrounding buildings, the instability of the tunnel inside, easy to cause the collapse of construction tunnels, endangering construction safety, so ensuring construction safety becomes the key to the project. By combining the examples of the Heyi River Tunnel in Quzhou, the project focuses on the safety measures of tunnel surrounding rock, and simulates tunnel excavation with BIM technology.
\end{abstract}

\section{Introduction}

With the development of economy, people are more and more perfect in the construction of infrastructure, and the construction of infrastructure is becoming more and more complicated. The shallow burying and undercutting method of mining method is increasingly used in subway and river-crossing tunnel projects. The shallow burying and undercutting method is convenient for the operation of the used machines and has low cost requirements, the cost of construction is greatly reduced, but the shallow burial method also has its own limitations: the shallow burying and undercutting method is likely to cause the settlement of the nearby ground surface to cause the instability of the tunnel and the collapse of the construction tunnel, which is dangerous to the construction safety.

Many scholars have conducted researches on the limitations of the shallow burying method in design and construction. Yi Xiaoming ${ }^{[1]}$ etc. conducted a mechanical analysis on the overall subsidence of the overlying stratum of the shallow buried tunnel through the analysis of the onsite monitoring data of urban tunnel construction, and believed that the overall subsidence and collapse of the overlying stratum of the tunnel was caused by the surrounding area. The result of the combined effect of the mechanical properties and geometric properties of the rock. Shi Yaxin ${ }^{[2]}$ etc. used the theory of random medium method to study the prediction of surface movement and deformation caused by the construction of large-section shallow buried tunnels, and obtained relevant prediction formulas for surface movement and deformation. Zhou Shunhua ${ }^{[3]}$ took the tunnel Based on in-situ observations and indoor centrifugal model tests, the scaffolding principle of the pipe roof method in the construction of shallow burial excavation in soft ground is studied. It is believed that the pipe roof mainly strengthens the surrounding rock and spreads the pressure of the surrounding rock, while reducing the excavation Relieve stress. Li Shuchen ${ }^{[4]}$ and others used fracture damage finite element and numerical software to study the minimum rock cover thickness of submarine tunnels, and established a displacement convergence method to determine the minimum rock cover thickness of submarine tunnels. In the 1970s, Lebuschwitz based on actual Observation of the tunnel failure phenomenon, proposed the tunnel rupture wedge theory ${ }^{[5]}$, which means that the deformation pressure is the main pressure of the deep tunnel.

The research on shallow burying and undercutting at home and abroad has become mature, but there are relatively few researches on shallow burying and undercutting under relatively special geological conditions. Because the shallow burying and undercutting tunnels are located under the Qujiang River, the The safety of tunnels digging across the river needs further study.

\section{Project overview}

\subsection{Project location}

The construction section of the underground excavation section of the Heyi River Crossing Tunnel is located below the Qujiang River. During the construction process, the tunnel excavation will cause uneven ground settlement, and the probability of tunnel collapse will be greatly deepened. In view of the safety problems in this project, the During the construction of the project, the surrounding rock and BIM simulation are carried out to ensure the safety of the construction. 


\subsection{Engineering scheme}

During the construction of soft soil layer tunnels, scientific researchers have conducted a lot of research on the soft soil layer tunnel construction method, structural stress system, settlement and deformation control, etc ${ }^{[6,7]}$.Heyi River Crossing Tunnel Crossing Qu Jiang Duan adopts the mining method of underground excavation. In order to reduce the impact of surface settlement during construction, the tunnel adopts a unidirectional single-hole section. Long pipe shed support method is used for grouting to pre-reinforce the surrounding rock to ensure the stability of the tunnel during construction. BIM simulates the construction law of shallow buried and undercut excavation to avoid instability risks during tunnel construction.

\section{Safety measures}

\subsection{Construction safety measures}

\subsubsection{Initial support}

The cross-river tunnel adopts one-way single-hole section. In order to strengthen construction quality control, sufficient support preparations must be made before construction. The initial support (Figure 1 initial support distribution map) adopts modified polyester The fiber $\mathrm{C} 25$ concrete spraying ensures that the support rigidity is in line with the actual situation and controls the settlement of the stratum and the surface ${ }^{[8]}$. To prevent the problem of ground subsidence, it is necessary to improve the soil to ensure the positive movement of the soil, use the long pipe roof support method for grouting, and pre-reinforce the surrounding rock to achieve the stability of the soil structure and avoid the large movement of the soil.

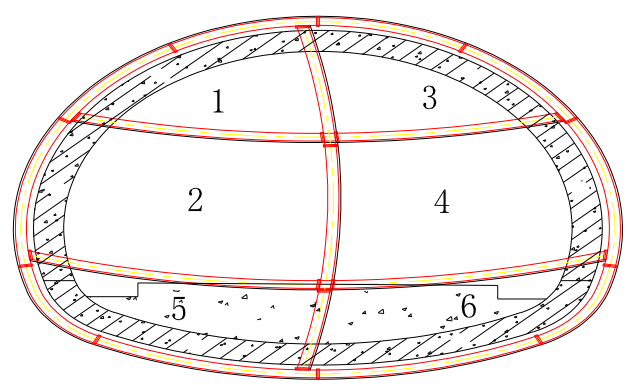

Fig.1. Initial support distribution map

\subsubsection{Secondary lining}

The secondary lining is made of waterproof concrete, sprayed with C35 reinforced waterproof concrete, and the arch, side wall, and invert are tested and found to be C35 reinforced waterproof concrete. After the surrounding rock and the initial support deformation become stable, the secondary lining is carried out to strengthen the stability of the lining structure and avoid collapse in construction. The initial support link focuses on the load bearing capacity of all structures, and the value of safety reserve is used in the secondary support. These two links need to carry out joint bearing on the structural load capacity. Support occupies a major position in the support to support the stability of the tunnel and avoid collapse.

\subsection{Application of BIM in engineering safety}

The comprehensive application of BIM auxiliary construction technology ensures that the foundation reinforcement achieves the desired effect, guarantees the tunnel construction quality, and effectively controls the project $\operatorname{cost}^{[9]}$.

\subsubsection{Deepening the design}

The process of engineering modeling for the passage across the river is a comprehensive "three-dimensional proofreading" process. During this process, a large number of problems hidden in the design can be found. These problems often do not involve specifications, but are closely related to professional cooperation, as shown in Figure 2. As shown, through the process of "threedimensional verification", safety threats in construction are avoided.

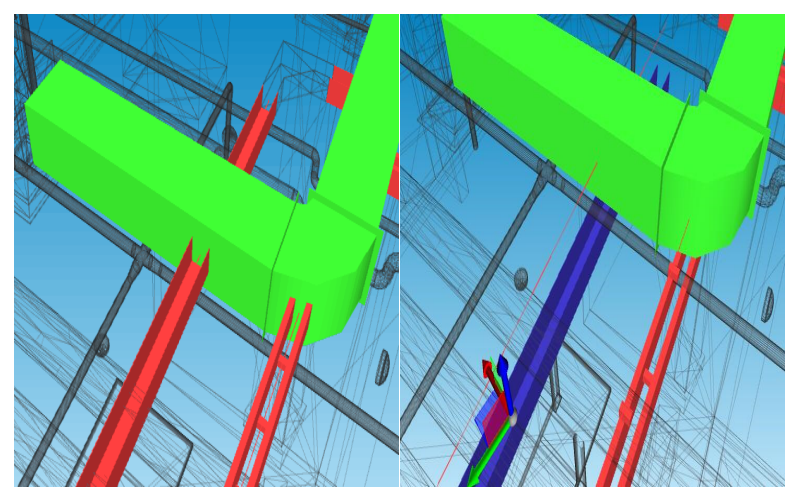

Fig. 2. BIM deepening design before and after comparison

\subsubsection{Simulation roaming and construction plan simulation}

With the help of 3D roaming, the tunnel can be displayed in three dimensions from the whole to the part, key nodes, and complex procedures, so as to improve the sensory perception of the various parts of the tunnel. Secondly, provide technical guidance to the construction party to avoid blind construction safety hazards. According to the design drawings and construction plan, establish a detailed model of the special technical plan and the special safety plan, simulate its construction method, identify unreasonable and problematic construction methods in the design, verify the interference and conflicts in the construction, and optimize the construction resources Configure, shorten the construction period, save costs, and ensure construction safety (Figure 3 simulation of the construction plan of bored piles). 


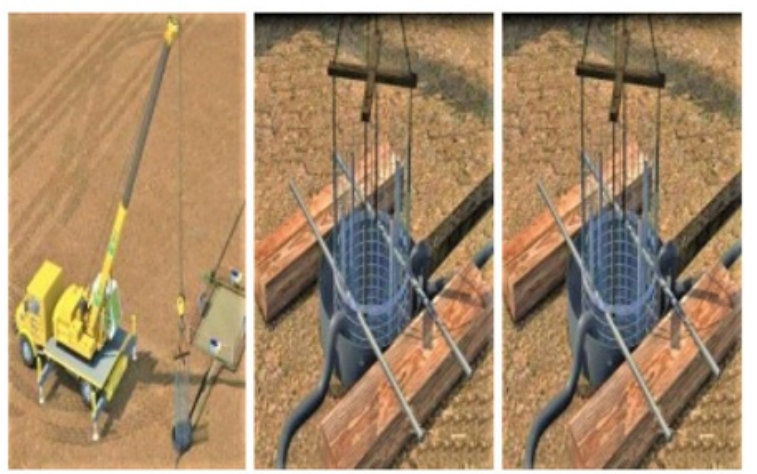

Fig.3. Simulation of the construction plan of bored piles

\subsubsection{Three-dimensional technical clarification}

The dynamic construction process is demonstrated with the aid of a three-dimensional visualization image, and the safety construction precautions and technical points are visually expressed, so that the construction personnel can more accurately understand the design intent, so as to better control the on-site construction quality. The video construction technology of the steel support of the buried section is explained as shown in Figure 4. After the civil structure is completed, through the application of threedimensional scanning technology, the required structural section point data can be quickly collected, and the theoretical and actual comparative analysis results can be quickly obtained through information technology (Figure 5). Through BIM technology, each stage and each key index , the current supply information of each organization, each specialty, and each project is no longer limited to adjacent members, ensuring the timeliness and interactivity of information. With the deepening and progress of engineering projects, the BIM information model is gradually deepened and refined, and continuously updated, revised and supplemented to ensure the integrity of the safety, quality, and inspection process results in the project construction process.

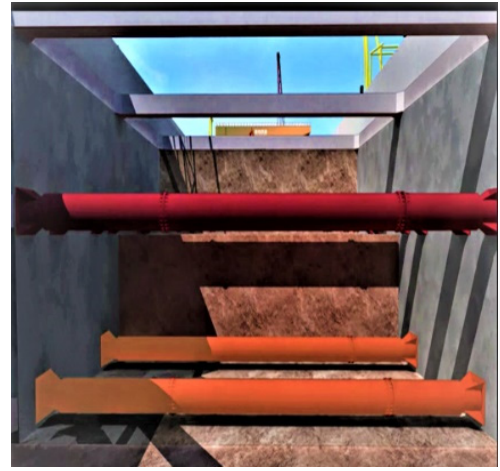

Fig.4. Video construction of steel support in open cut and buried section

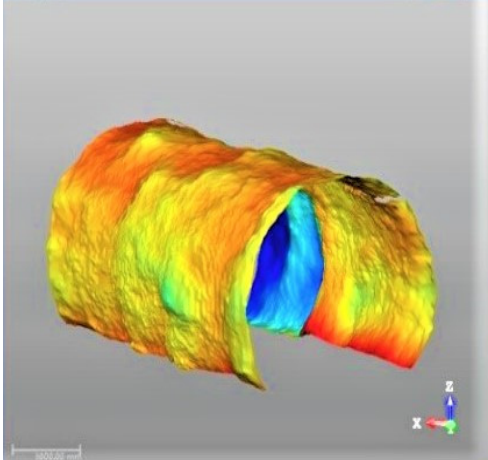

Fig.5. Tunnel status scan

\section{Conclusion}

The engineering geological environment of the rivercrossing tunnel is complex and the risk of underwater digging is relatively high. The safety guarantee is particularly important. During the construction, the tunnel is prone to collapse due to surface settlement, which affects the safety of tunnel construction. In this paper, combined with the construction of Heyi Project, the surrounding rock is supported by long pipe sheds and secondary lining, which can effectively achieve the stability of the soil structure and avoid the large-scale movement and collapse of the soil. The application of BIM technology in the project can effectively avoid construction risks and ensure the safe progress of the project.

\section{References}

1. YI Xiaoming, Zhang Dingli, Pang Tiezheng \& Luo Jianjun. (2009). Mechanical mechanism analysis of the overall subsidence of the overlying stratum of an urban tunnel. Chinese Journal of Rock Mechanics and Engineering (S1), 2860-2867.

2. SHI Yaxin, Tao Dejing, Wa Mingnian . Surface movement and deformation prediction caused by the construction of shallow buried tunnels $[\mathrm{J}]$. Rock and Soil Mechanics,2008,29( 2) : 465-474 .

3. ZHOU Shunhua. (2005). Scaffolding principle of pipe roof method in construction of shallow buried and undercut in soft ground. Chinese Journal of Rock Mechanics and Engineering (14), 2565-2570.

4. LI Shucai, Li Shuchen, Xu Bangshu, Wang Hanpeng \& Ding Wantao. (2007). Research on the method for determining the minimum rock cover thickness of subsea tunnels. Chinese Journal of Rock Mechanics and Engineering (11), 2289-2295.

5. YU Xuefu, ZHENG Yingren, LIU Huaiheng, et al. Stability analysis on surrounding rocks of underground works[M]. Beijing: Coal Industry Press, 1983: 245-259.

6. DONG Yunpeng \& Sun Xiaojing. (2018). Research on the impact of small clear distance subway tunnels 
through buildings at short distances. Journal of Railway Engineering Society (11), 87-91.

7. Xi Shujuan \& Huang Lin. (2015). Research on the stability of the tunnel underneath the existing building with small clear distance. Railway Standard Design (06), 117-122.

8. LUO Ganxiao. (2019). Shallow buried tunnel construction technology and its ground subsidence control strategy. Housing and Real Estate (03), 157

9. WANG Zhijun. (2018). Application of Shallow Buried Excavation Method in Tunnel Construction Technology. Transportation World (21), 136-137. 